C'est pourquoi nous avons, de notre côté, cherché un autre procédé d'évaluation et voici la technique à laquelle nous nous sommes provisoirement arrêté :

Technique. - On pèse exactement un gramme de caséine préalablement pulvérisée au moulin à café, de façon à obtenir toujours un grain identique à lui-même. Introduire la quantité pesée dans une capsule de platine bien propre (une capsule de nickel peut suffire) et mettre macérer avec $10 \mathrm{~cm}^{3}$ d'eau distillée, en chauffant légèrement et en agitant avec un agitateur de verre. Laisser déposer la easéine et décanter le premier liquide dans un tube à essai. Ajouter $10 \mathrm{~cm}^{3}$ d'eau distillée sur la caséine et répéter la même opération en recueillant le liquide décanté dans le même tube à essai.

Renouveler une troisième fois la même opération. Ajouter au liquide trois gouttes de phtaléine du phénol et titrer avec la soude Dornic.

Expression des résultats. - Nous avons toujours obtenu le même résultat pour la même caséine. C'est la condition nécessaire et suffisante pour permettre une comparaison.

Supposons qu'on ait trouvé $12^{\circ}$ Dornic. On rapportera le résultat à 100 gr. de caséine en disant que cette caséine renferme 1 gr., 2 d'acide lactique pour cent. S'il s'agissait d'une easéine aux acides, on pourrait exprimer le résultat en acide sulfurique en multipliant $1 \mathrm{gr}, 2$ par 0,54.

Dans le cas d'acide ehlorhydrique, il suffirait de multiplier 1 gr. 2 par 0,41 .

Résultats. - En employant cette technique, les caséines ẩ la présure donnent des résultats extrêmement faibles : $1 / 10$ de centimètre cube de soude Dornic suffit à faire rougir la phénolphtaléine.

Quant aux caséines lactiques, l'acidité est essentiellement variable en moyenne de 6 à $12^{\circ}$ Dornic. Ces résultats varient évidemment avec la plus ou moins grande perfection des opérations de lavage.

\title{
ANALYSE DES CASÉINES INDUSTRIELLES
}

\section{par André CHOLLET,}

Ingénieur Agronome, Professeur à 1'Ecole de Laiterie de Surgères

Nous nous contenterons de rappeler les méthodes que nous employons au laboratoire de l'Ecole de Laiterie de Surgères (ces méthodes avaient été mises au point par DoRNIC et DAIRE qui les avaient publiées en 1909, dans la Revue Générale du Lait, p. 328) seule la méthode de dosage de l'acidité que nous n'employons que depuis deux ans a été exposée dans Le Lait, 1928, page 21.

Prélèvement de l'échantillon. - Il est nécessaire de prélever un échantillon moyen sur le contenu de plusieurs sacs d'une même marchandise, et en différents points d'un même sac ; les cannes-sondes employées dans le commerce des grains sont très utiles dans ce but. La caséine étant douée 
de propriétés hygroscopiques prononcées, la quantité d'eau varie souvent dans un même sac de l'intérieur à l'extérieur; de plus, même dans une même usine la composition n'est pas toujours la même, d'un jour à l'autre. Il est évident que les variations sont encore plus grandes quand il s'agit d'un mélange de caséines de provenances différentes.

Dosage de l'humidité. - C'est le dosage le plus fréquent, l'acheteur ayant intérêt à ce que la caséine soit la plus sèche possible, le vendeur au contraire, à ce que la teneur en eau soit aussi élevée qu'il se peut. A l'heure actuelle, on admet que la caséine ne doit pas contenir plus de $12 \%$ d'eau.

La dessiccation de la caséine en grumeaux est une opération très longue; elle n'est pas terminée après 8 heures à $105^{\circ}$ ou après 12 heures à $100^{\circ}$. D'autre part, dans les caséines réduites en farinés trop fines la dessiccation peut être gênée par la formation d'une croûte à la surface (dans ce cas on met dans la capsule une petite baguette de verre avant de faire la tare, baguette qui sert à briser la croute de la caséine quand elle se produit).

La forme qui convient le mieux pour la dessiccation est celle du gruau ou de la semoule; on l'obtient au laboratoire en broyant les grumeaux de caséine dans un moulin à café. La dessiccation s'effectue à 100-1020 pendant 5 heures en mettant dans une capsule de porcelaine, séchée et tarée, 5 grammes de caséine. Pour les caséines très humides, il est prudent de remettre à l'étuve pendant une-demi heure et de peser à nouveau; on ne considèrera la dessiccation comme terminée que si la différence entre les deux dernières pesées ne dépasse pas un ou deux milligrammes.

Dosage de la matière grasse. - C'est une application de la méthode Schmid-Bondzynski.

Dans une fiole conique de $50 \mathrm{cc}$. environ préalablement séchée et tarée, on met 3 grammes de caséine broyée. On ajoute $10 \mathrm{cc}$. d'acide chlorydrique de poids spécifique 1,125. On chauffe sur un bec Bunsen en protégeant par une toile d'amiante. La caséine se dissout complètement et on continue à chauffer pendant 8 à 10 minutes, en maintenant une ébullition très modérée et en évitant les projections. On laisse refroidir et on verse le contenu dans un des tubes utilisés pour le dosage $\mathrm{du}$ lait suivant la méthode de Rose Gottlieb. On rince les parois du vase conique avec 25 centimètres cubes d'éther sulfurique ajouté par petite portion. Cet éther est versé dans le tube. On bouche le tube, et on agite fortement. On recommence avec $25 \mathrm{cc}$. d'éther de pétrole bouillant audessous de $70^{\circ}$ que l'on introduit dans le tube, on bouche, on agite et on abandonne au repos. Après deux heures de repos, on peut siphonner la majeure partie de la couche éthérée dans une fiole conique d'environ 100 cc. préalablement séchée et tarée. On évapore le plus possible des éthers au bain-marie et on achève la dessiccation à l'étuve à $100^{\circ}$ pendant 
5 heures. Avec un peu d'habitude, on arrive à siphonner la presque totalité de la couche éthérée, ce qui dispense de l'emploi de tubes gradués.

Dosage des cendres. - Lincinération se fait sur 3 grammes de caséine dans une eapsule de platine tarée et recouverte au début pour éviter les pertes dues aux projections ou au boursouflement. On ne dépasse pas le rouge sombre et on arrête l'opération quand les cendres sont blanches. L'opération est relativement rapide avec les caséines à la présure; elle est beaucoup plus lente avec les easéines lactiques.

\section{NOTE SUR LE DOSAGE DE LA MATIËRE GRASSE DANS LA CASÉINE}

\section{par Mare FOUASSIER,}

En ce qui concerne le taux de matière grasse dans les caséines, il est indispensable qu'une entente internationale intervienne. Beaucoup de marchés pour l'exportation reposent sur ce taux, et les méthodes utilisées pour le déterminer ne donnent pas des résultats concordants.

En France, on semble s'en tenir à la méthode par épuisement avec des solvants appropriés, en utilisant la Soxhlet. Cette méthode est longue et son résultat toujours inférieur à celui que l'on devrait obtenir réellement. La grosseur du grain, la pénétration aux solvants en font une cause d'insuccès.

Je signale pour mémoire le broyage au sable de la caséine préalablement humidifiée ; là encore, l'extraction est incomplète.

Seules, les méthodes basées sur la désagrégation préalable ét totale du grain de caséine, dans un milieu acide ou alcalin, suivie de l'extrac. tion de la matière grasse de ce milieu, par les solvants appropriés, permettent le dosage certain et régulier de la totalité de la matière grasse d'une caséine.

En Allemagne, on utilise la méthode Gottlieb-Ratzlaff, ou la méthode Schmid-Bondzynski-Ratzlaff, l'une et l'autre utilisant la désagrégation en milieu acide. La dernière de ces méthodes est décrite dans les Annales des Falsifications et des Fraudes, no 127 , page 41 , où elle est appliquée aux fromages.

Appelé à faire de nombreuses analyses de caséines destinées à l'exportation, j'utilisais pour le dosage de la matière grasse, la méthode par extraction au Soxhlet, mes résultats étaient toujours très sensiblement différents de ceux obtenus en Allemagne, par exemple, lors de la contreanalyse, ce qui a donné lieu à de multiples contestations. Il m'est apparu nécessaire de demander communication de la méthode utilisée, et par suite, de l'employer moi-même, il en est résulté une complète concordancenentre les résultats obtenus de part et d'autre et, par conséquent, la disparition de toute discussion. La méthode indiquée est celle de Gottlieb-Ratzlaff. 